\title{
Foro Experimental sobre Recursos de Matemática Aplicada a las Telecomunicaciones. Utilización de las Tecnologías de la Información y la Comunicacion en la docencia.
}

\author{
Salvador Merino Córdoba \\ smerinoluma. es \\ Ángel Mora Bonilla \\ amoralctima.uma.es \\ María Luz Muñoz Ruiz \\ munoz@anamat.cie.uma.es \\ José Manuel González Vida \\ vidalanamat.cie.uma.es \\ Javier Martínez del Castillo \\ javimectima.uma.es \\ Enrique Mérida Casermeiro \\ merida@ctima.uma.es \\ Pablo Cordero Ortega \\ pcorderoluma.es \\ Francisco J. Rodríguez Sánchez \\ fjrodriectima.uma.es \\ José Luis Galán García \\ jl galan@uma.es \\ Pedro Rodríguez Cielos \\ prodriguezluma.es \\ Yolanda Padilla Domínguez \\ ypadilla@ctima.uma.es \\ Gloria Gutiérrez Barranco \\ gloriagbectima.uma.es \\ Universidad de Málaga
}

\section{Introducción}

En este artículo describimos una experiencia a la que hemos denominado FERMAT (Foro

Experimental sobre Recursos de Matemática Aplicada a las Telecomunicaciones) y que se lleva realizando en la Escuela Técnica Superior de Ingeniería de Telecomunicación de la Universidad de Málaga desde hace dos años.

Este trabajo se ha encauzado bajo los siguientes proyectos:

- El Proyecto de Innovación Educativa Proyecto FERMAT (curso 04/05. Universidad de Málaga, código PIE 04/030).

- El Proyecto Andaluz de Formación del Profesorado Universitario FERMAT (curso 04/05. 
FERMAT es un foro de trabajo desarrollado por un grupo de doce doctores, profesores del Departamento de Matemática Aplicada de la Universidad de Málaga. Durante los dos últimos cursos se ha convertido en el punto de encuentro para alumnos y docentes de asignaturas de Matemáticas tan diversas como los Métodos Numéricos, Álgebra, Cálculo, Matemática Discreta, Análisis Vectorial y Ecuaciones Diferenciales o Ampliación de Matemáticas. En el último año se han producido más de 1.000.000 accesos y 70.000 visitas, lo que evidencia el éxito de este proyecto y la necesidad de realizar experiencias de este tipo.

\section{Objetivos y Metodología}

El problema originario era tratar de paliar el fracaso (tanto a nivel académico como de implicación de los alumnos en su desarrollo) que existía en la asignatura Métodos Numéricos de las distintas especialidades de la titulación de Ingeniería Técnica de Telecomunicación de la Universidad de Málaga (800 alumnos). Esta asignatura consta de 4.5 créditos y se imparte en dos contextos diferentes: el primero mediante clases teóricas, apoyadas en tecnologías multimedia, en la que se introducen los conceptos básicos y se resuelven problemas con tamaño reducido; el segundo en clases de laboratorio, donde se enseña al alumno a enfrentarse con problemas reales de un tamaño que sólo puede ser abordado mediante el empleo del ordenador y con el uso de un software específico (Matlab).

Para solucionar este problema, nos marcamos como objetivo principal el desarrollo de un entorno educativo que complementara la formación presencial tradicional.

Tras los buenos resultados obtenidos, ese trabajo fue la base de la adaptación de la docencia de las diferentes asignaturas a las que antes hacíamos mención. Además, hemos pretendido ir adaptándonos a las nuevas directrices dadas en la declaración de Bolonia en cuanto a facilitar la individualización y tutorización, presencial o no, de la enseñanza universitaria.

\section{Descripción del portal FERMAT}

A continuación pasamos a comentar el funcionamiento general del portal FERMAT y las características principales de sus diferentes secciones.

\subsection{Presentación}

La ilustración 1 muestra la página de inicio de FERMAT. En ella se recogen las diferentes asignaturas con que cuenta el Departamento de Matemática Aplicada de la Universidad de Málaga en la Escuela Técnica Superior de Ingeniería de Telecomunicación. También podemos observar las cuatro secciones principales con que cuenta el portal y todos los links de interés (en su parte derecha) que pueden servir de ayuda en el desarrollo de las asignaturas de Matemáticas y en la realización de ejercicios y cuestionarios. 


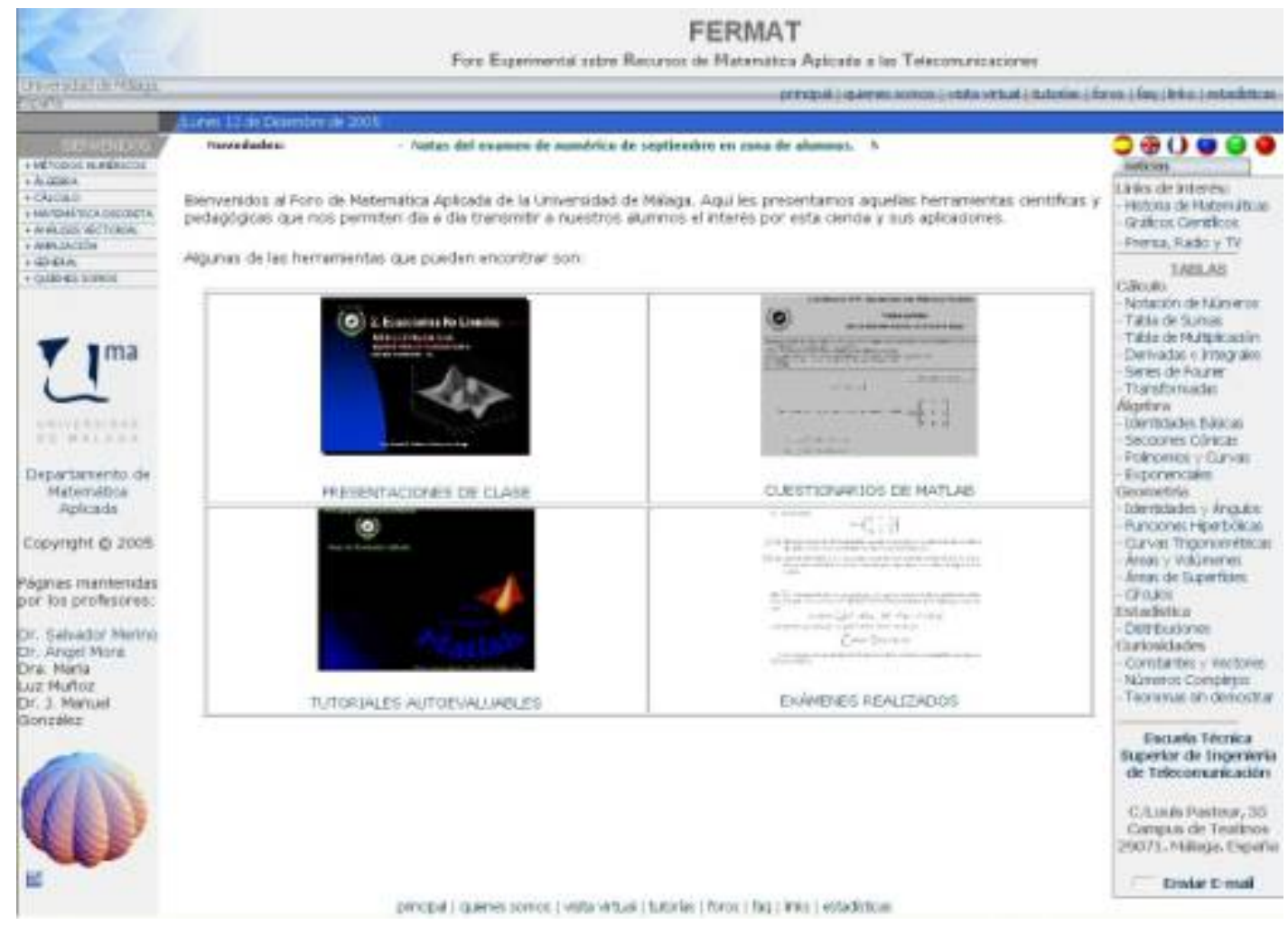

Ilustración 1. Página de inicio de FERMAT

\subsection{Menús Desplegables}

Siguiendo la estructura de árbol, cada asignatura cuenta con los diferentes niveles (carrera técnica o superior), su esquema, presentaciones, etc.

\begin{tabular}{|c|c|c|}
\hline + MÉTCDOS RUMÉRICOS & & \\
\hline \multicolumn{2}{|l|}{ + ALGEERA } & \\
\hline + chalcuso & + INGERIERLA TÉCHICA & \\
\hline + MATEMǴTICA DISCRETA & + DNGERTERTIA SUPERICR & > ESQUEMA \\
\hline \multicolumn{2}{|l|}{ + ARALLSSIS VECTOKLAL } & - pQESENTACICNES \\
\hline \multicolumn{2}{|l|}{ - AMPLLACICAR } & >FORO \\
\hline \multicolumn{2}{|l|}{ + GENERAL } & $\rightarrow F A Q$ \\
\hline \multirow[t]{3}{*}{ + QUIENES SCHOS } & & $\rightarrow$ 2OHA DE ALLMEVOS \\
\hline & & - CALENDMRTO \\
\hline & & DESTADISTICAS \\
\hline
\end{tabular}

Ilustración 2. Estructura de árbol

\subsection{Visita Virtual}




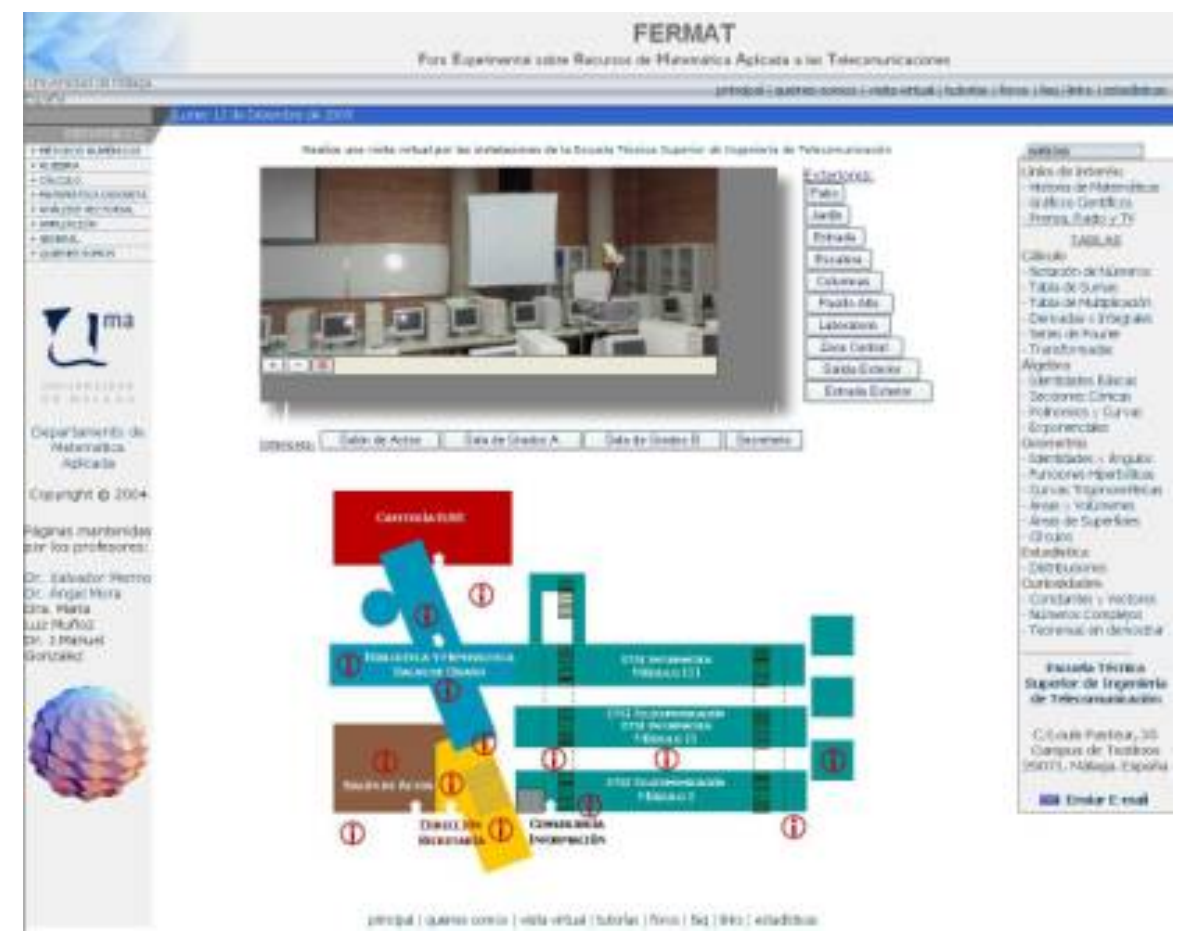

Ilustración 3. Visita Virtual

Al igual que a través de los menús y links, el alumno puede acceder a las diferentes secciones del portal como si fuera paseando por el centro. Se irá encontrando con iconos y puertas que le permiten acceder a las instalaciones de la Escuela y desde sus aulas y laboratorios puede entrar en las diferentes presentaciones de las asignaturas, exámenes, tutorías, notas, paneles de noticias, biblioteca, etc.

\subsection{Esquema de las asignaturas}

Cada una de ellas puede contar con diferentes secciones como las que se observan en la ilustración 4.

En las presentaciones se accede a los diferentes temas de la asignatura realizados básicamente en los entornos de Power Point y Acrobat Reader. Estas presentaciones son las utilizadas por el profesorado en clase y contienen todos los fundamentos teóricos y los ejemplos de aplicación de cada asignatura.

En los cuestionarios, realizados con Hot Potatoes, se resuelven por parte del alumno cuestiones acerca de la asignatura y del manejo del software especializado que cada una necesita.

Los exámenes han sido elaborados con LaTeX2ع y se van colocando conforme se van realizando y evaluando.

Los tutoriales, realizados con aplicaciones propias de proyectos fin de carrera, interconectan LaTeX2 $\varepsilon$ y Adobe Acrobat para producir manuales autoevaluables que se van siguiendo en los laboratorios. En ellos encontramos el desarrollo de los programas MatLab, Derive o Mathematica (depende de la asignatura) junto con preguntas tipo test para evaluar el nivel de conocimientos adquirido.

Por último, la bibliografía esta directamente interconectada con el catálogo "Jabega" de la Universidad de Málaga. Así, al pulsar sobre cualquier libro, los alumnos acceden a ver en qué biblioteca se encuentra, de cuántos volúmenes disponen y cuándo pueden solicitarlo. 


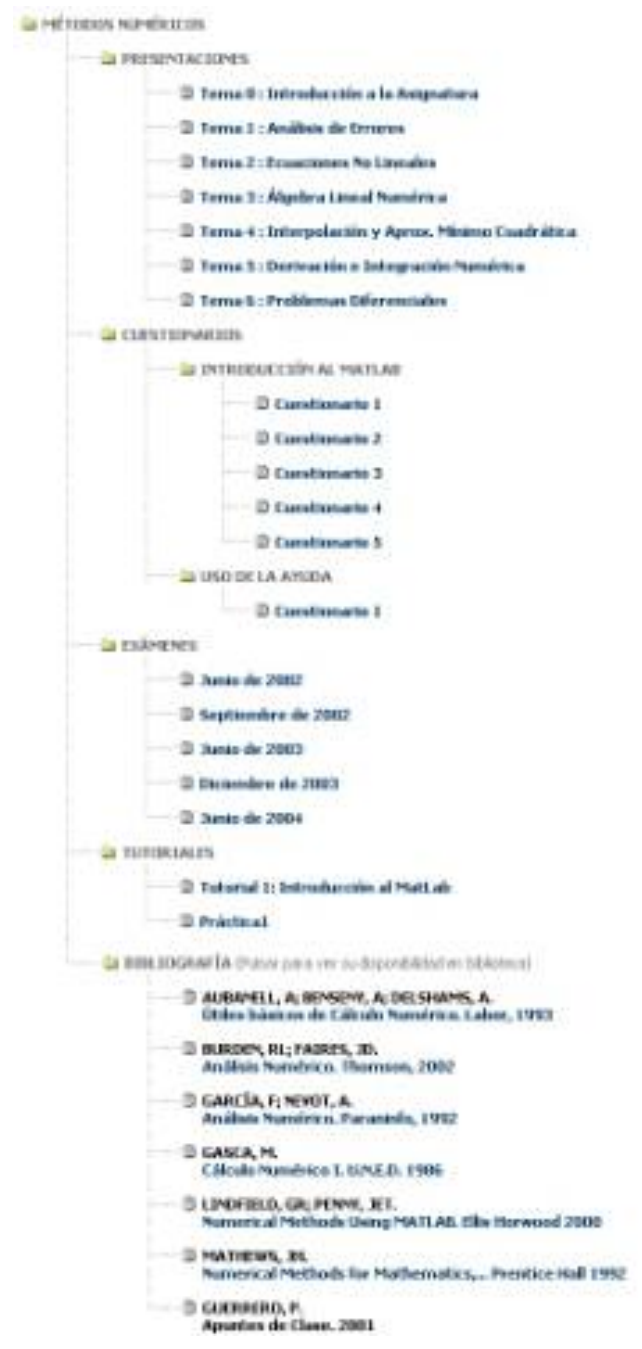

Ilustración 4. Esquema

Algunas imágenes de los conceptos aquí vistos son las siguientes:
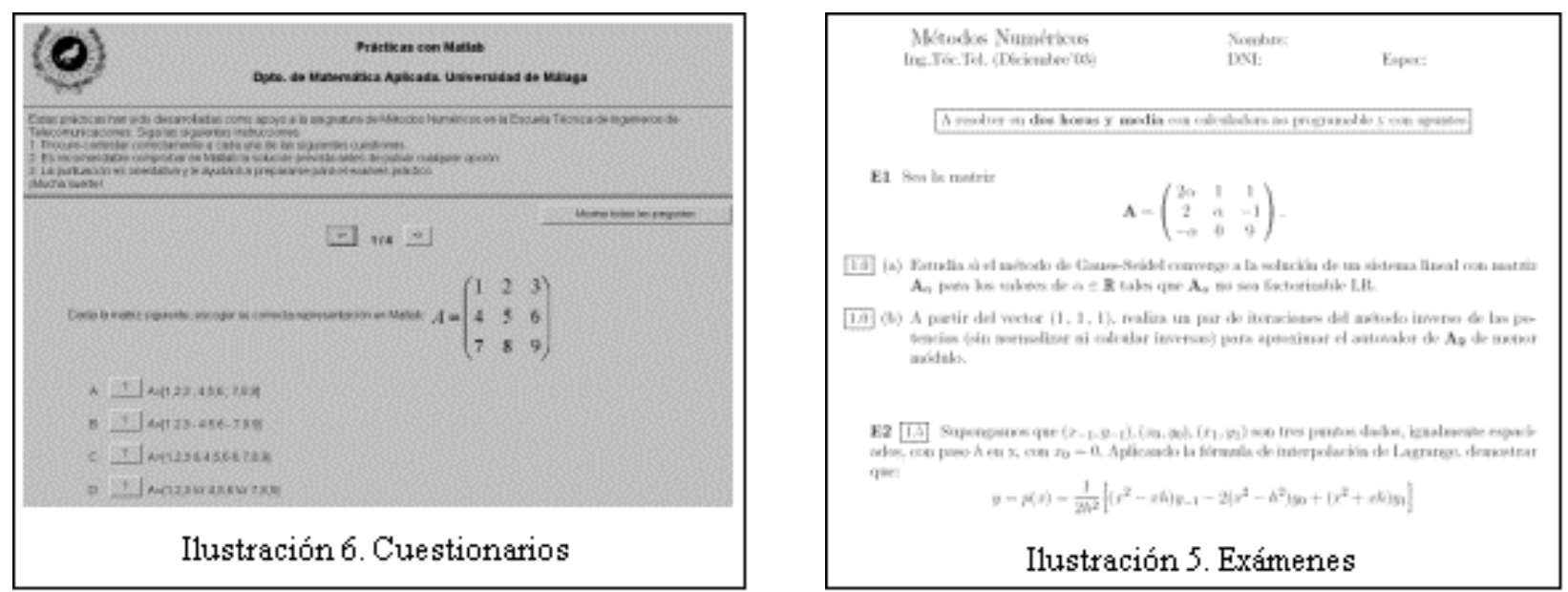

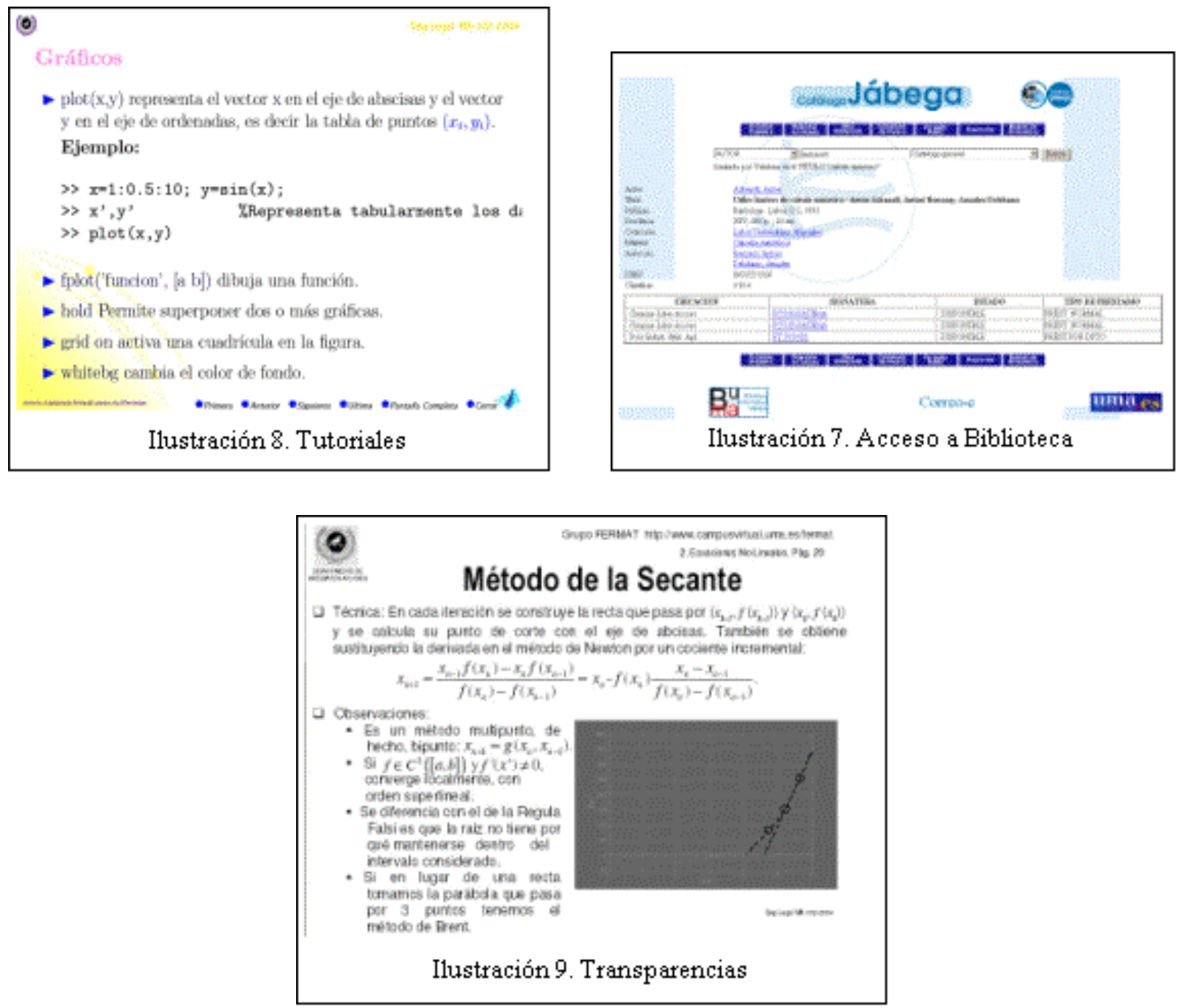

\subsection{Herramientas Complementarias a cada asignatura}

FERMAT cuenta con diversas herramientas para el seguimiento de las asignaturas que se interconectan con el Campus Virtual de la Universidad de Málaga. Entre ellas cabe destacar:

\section{El calendario}

Con él los alumnos tienen un seguimiento de prácticas, exámenes y cambios en la asignatura.

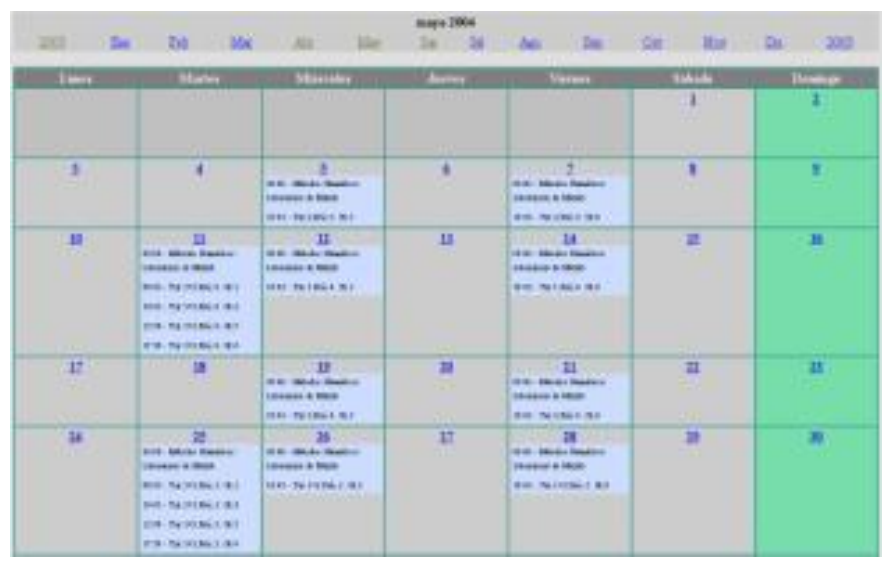

Ilustración 10. Calendario

\section{Foros}

Dirigidos a la participación de los alumnos y a la interrelación no presencial entre ellos y el docente. 

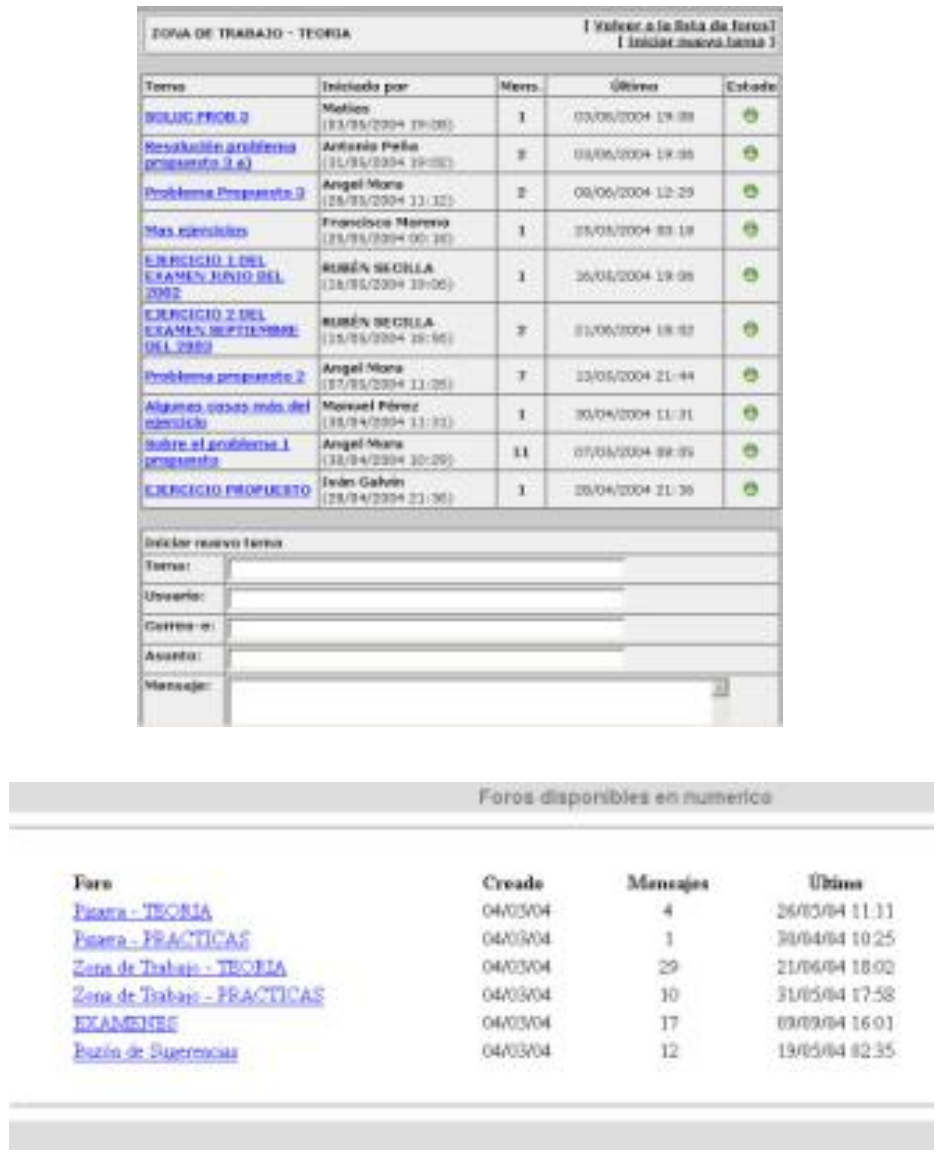

Ilustración 11. Foros

\section{Zona de alumnos}

Destinada a contener todo el material necesario para el seguimiento de las asignaturas. Su entrada se realiza bajo clave personal y en ella los alumnos pueden ver sus calificaciones, recoger el material permitido en los exámenes, conseguir los manuales del software o seguir el desarrollo teórico de la asignatura por internet.

\section{Index of/numerico/_alumnos/Práctica}

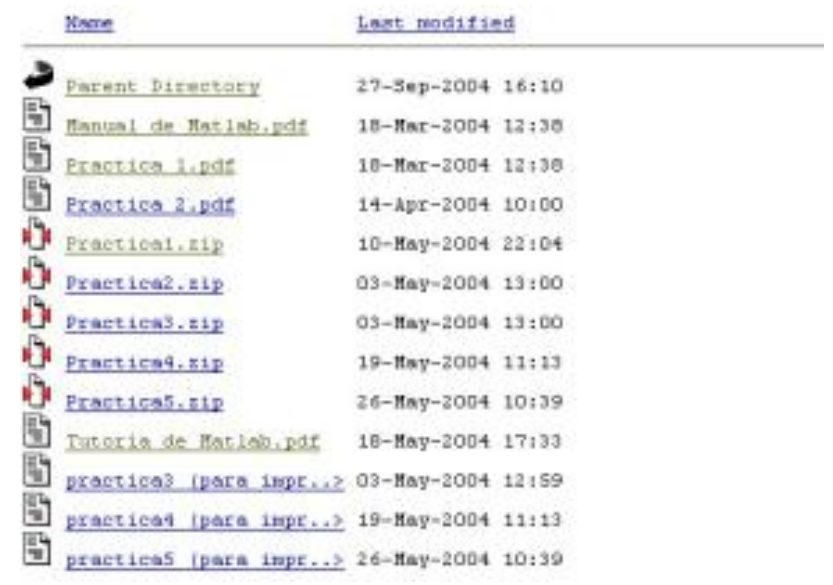

Ilustración 12. Zona de alumnos

\section{Estadísticas}

Con ellas podemos evaluar el acceso que se produce al portal y a cada una de las asignaturas que lo conforman, deduciendo de las mismas su estacionalidad, lugar de origen, cantidad de material que se recoge, páginas más visitadas, etc. 


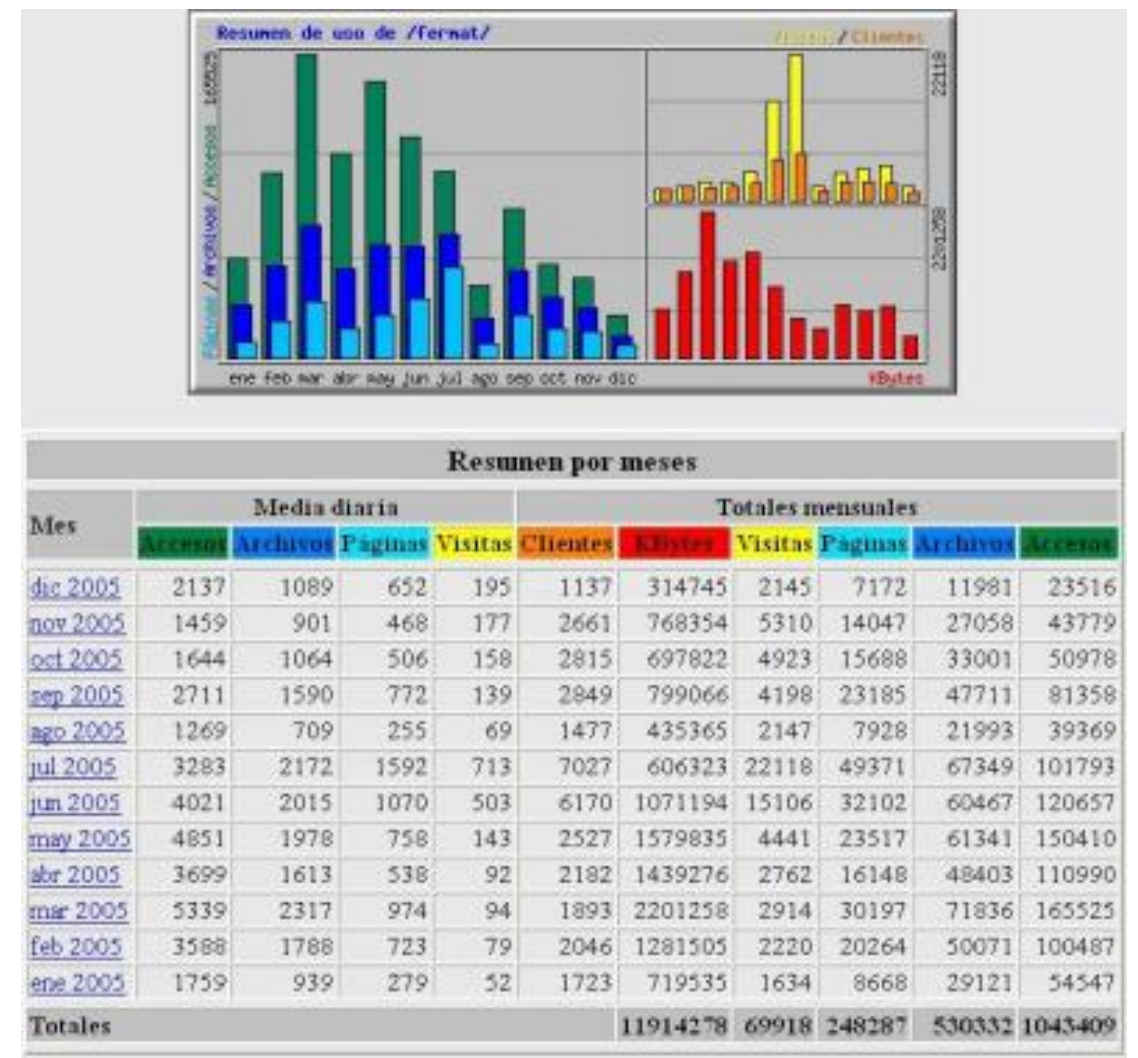

\section{Ilustración 13. Estadísticas}

\section{Encuestas de Calidad}

Realizadas al finalizar el desarrollo de las distintas asignaturas nos permiten ver aquellas lagunas existentes y recoger las recomendaciones del alumnado para ir mejorando día a día. Su resultado es directamente tabulado a través de la página y llega de forma numérica (a excepción de las recomendaciones) al responsable de cada asignatura.

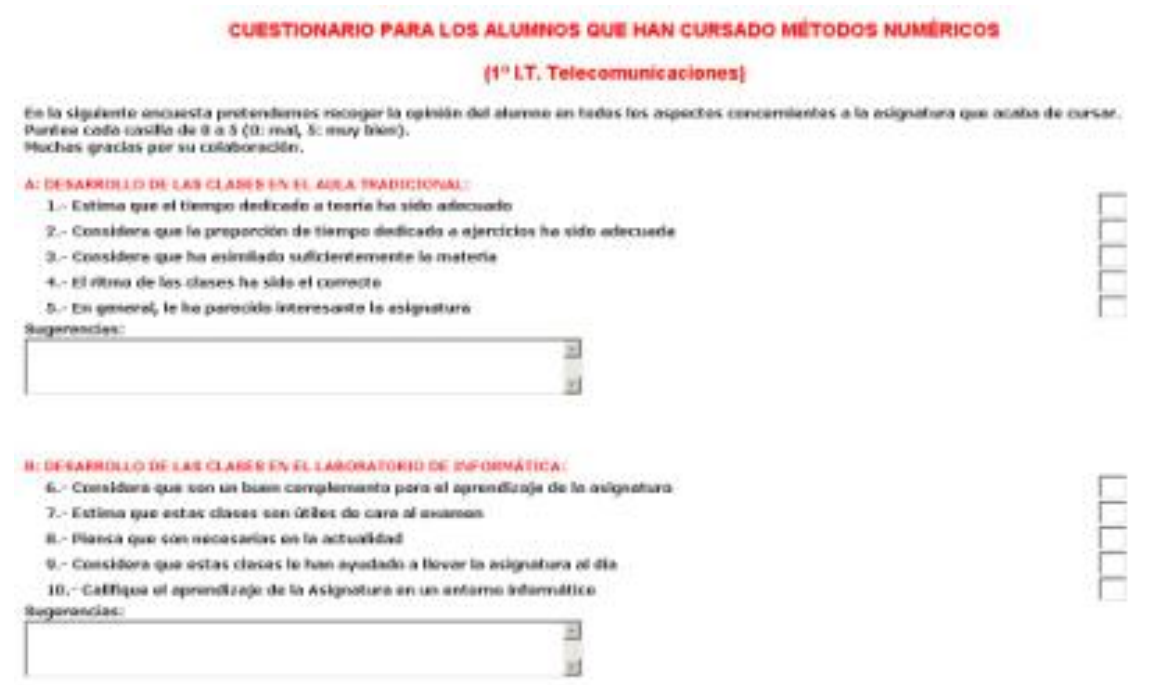

Ilustración 14. Encuesta de Calidad

\section{Tutorías}

En ellas se reflejan los horarios establecidos para realizar las tutorías presenciales de las asignaturas. 


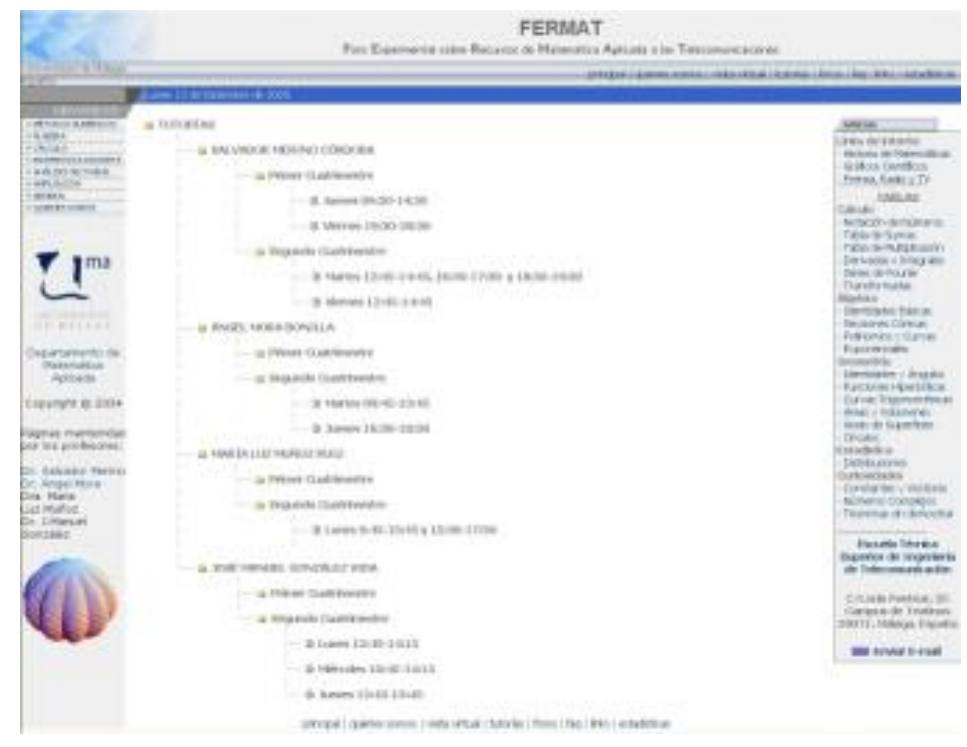

Ilustración 15. Tutorías

\subsection{Herramientas auxiliares}

Junto con el seguimiento de las asignaturas, y dentro de lo que consideramos adecuado para el conocimiento de las ciencias y el desarrollo integral de los universitarios, incluimos las siguientes herramientas.

\section{Gráficos científicos}

Estos gráficos científicos, extraídos del diario El Mundo y realizados en formato Flash, han sido elegidos para complementar las asignaturas de Matemáticas y ver su aplicabilidad en los diferentes campos de la ciencia.

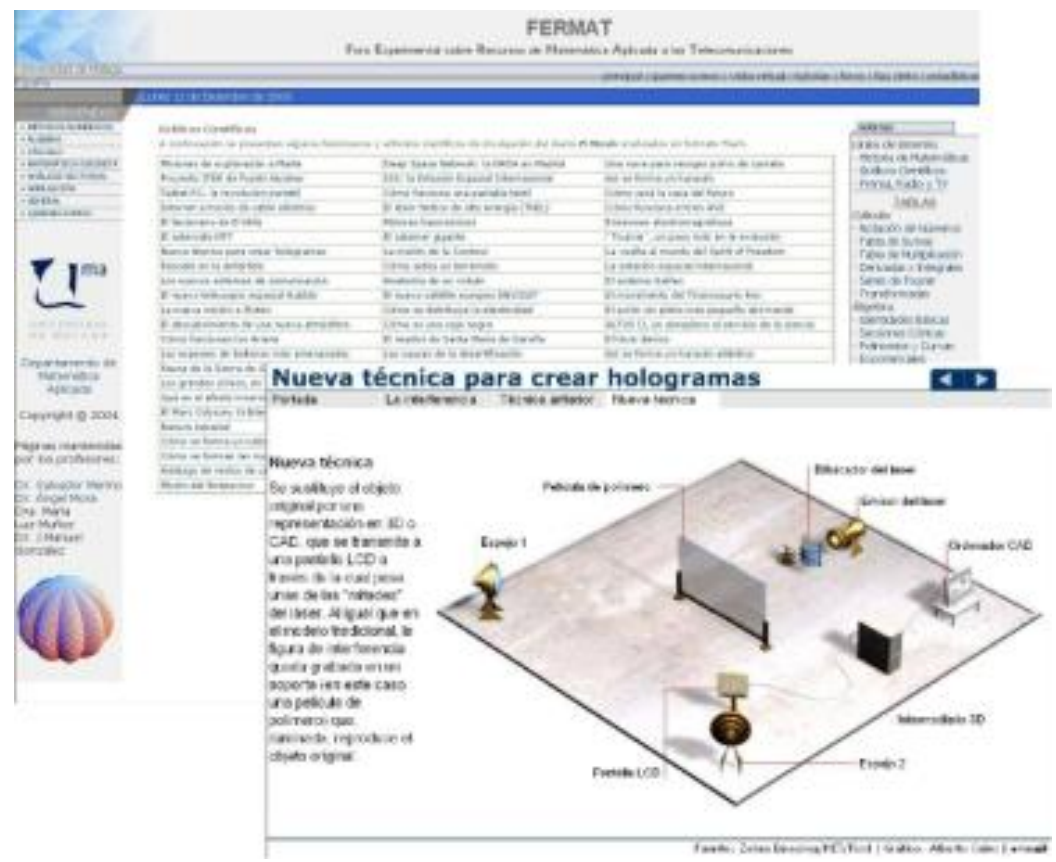

Ilustración 16. Gráficos científicos

Especialmente se ha hecho referencia a aquellos gráficos interactivos relacionados con el campo de las telecomunicaciones y de la informática, junto con los nuevos avances tecnológicos que se van sucediendo. 
Web creada en la Universidad de St.Andrews y donde recoge la historia de los principales matemáticos que han existido, sus descubrimientos y las curvas y fórmulas más destacadas.

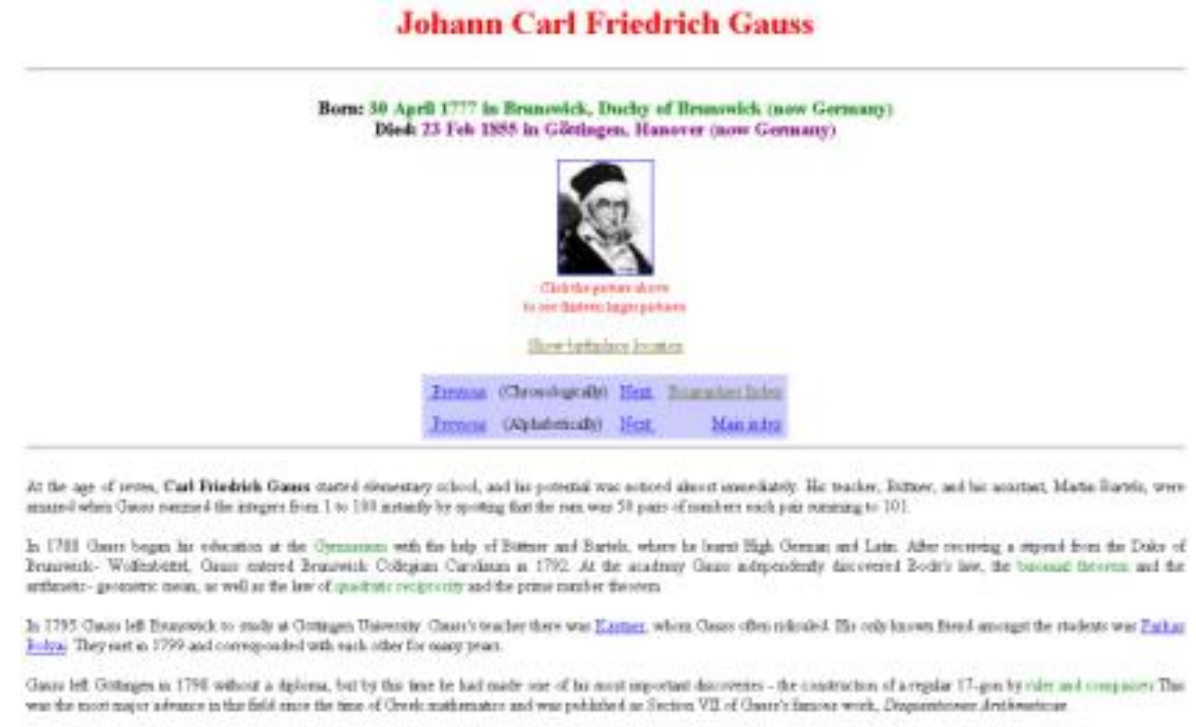

Ilustración 17. Historia de las Matemáticas

\section{Prensa, Radio y Televisión}

Estos links tratan de cubrir el mayor número de diarios tanto digitales como escritos, junto con una amplia variedad de televisiones y radios.

\section{Resultados obtenidos}

La experiencia realizada en estos dos primeros años de implantación nos ha permitido observar una mejora importante en el quehacer diario de las asignaturas. Entre estos resultados cabría destacar:

Mejor coordinación entre el profesorado que imparte las diferentes disciplinas y un equilibrio de niveles y de comunicación de conceptos al alumnado.

Mayor sensación de comodidad y facilidad de transmisión en las asignaturas por parte de los docentes, mejorando el trato humano y las relaciones profesor-alumno.

Incremento en la participación del alumnado, tanto en el número de los que siguen a diario las enseñanzas en el aula como los que participan por internet en los foros y debates que se proponen.

Mejora de la calidad de las prácticas en el laboratorio, con un importante apoyo multimedia en el desarrollo de los tutoriales autoevaluables de MatLab y su posterior aprendizaje y ensayo en los cuestionarios de los que dispone los alumnos en la red.

Cercanía a los centros de enseñanza mediante el empleo de visitas virtuales donde se introducen en las aulas, observan las presentaciones realizadas por los profesores, recogen el material de los laboratorios, ven el panel de notas y descargan las noticias de interés o comprueban los horarios de tutorías presenciales de cada profesor.

Utilización habitual de los enlaces directos entre las bibliografías de las asignaturas y el banco de datos de la biblioteca general de la Universidad de Málaga.

\section{Conclusiones}

Como resumen, podemos afirmar que FERMAT ha surgido para apoyar la enseñanza de las Matemáticas en la docencia universitaria y la formación integral y universal del alumnado. Al ser muy 
complicado manejar todo tipo de notación matemática ha sido necesario optimizar el modo de representar y manipular esta información con las herramientas actuales que las nuevas tecnologías ofrecen.

\subsection{Resumen de herramientas desarrolladas}

FERMAT cuenta actualmente con las siguientes herramientas:

Presentaciones que resumen de forma interactiva los conceptos esenciales de todos los temas de las asignaturas y que son utilizados por los alumnos vía web en su trabajo personal y por los docentes en sus exposiciones.

Tutoriales interactivos que ayudan a la formación presencial para el aprendizaje de las asignaturas y en los que se presentan cuestiones que el alumno va contestando, comprobando así su nivel de asimilación de conocimientos.

Cuestionarios relativos a la parte práctica de las asignaturas y que ofrecen al alumno de forma no presencial la posibilidad de auto-evaluar su proceso de aprendizaje.

Foros en los que los alumnos y profesores se encuentran para ayudar a resolver los ejercicios y las prácticas de las asignaturas. Los profesores proponen problemas y fomentan, valoran y evalúan la participación de los alumnos en dichos foros.

Calendario en el que se informa a los alumnos de la temporalización de las asignaturas así como de los posibles cambios que pueden producirse.

Referencias bibliográficas donde se accede a la disponibilidad en biblioteca de los textos recomendados en cada asignatura.

Visita virtual donde se realiza un paseo virtual por la Escuela de Telecomunicaciones y se accede a los laboratorios, clases, biblioteca, foros, notas, tutorías, información universitaria, etc.

Complementos que contienen toda aquella información que puede ser de utilidad al universitario, como:

o Presentación del Foro en Inglés, Francés, Árabe o Ruso, para acercar el uso de FERMAT a los no hispanohablantes que cada día suponen un porcentaje mayor de alumnos en nuestros centros.

o Gráficos interactivos sobre cuestiones científicas de actualidad.

o Acceso a los principales medios internacionales de prensa, radio y televisión en cualquier idioma.

o Conexión con la Historia de las Matemáticas y con las principales tablas y elementos de cálculo necesarios en las cuestiones propuestas en FERMAT.

\subsection{Trabajo Futuro}

Como trabajo futuro nos planteamos:

Mejorar la enseñanza de las diversas asignaturas utilizando nuevas tecnologías con mayor conexión a las herramientas pedagógicas.

Investigar la aplicación de las técnicas actuales de Enseñanza Asistida por Ordenador a la enseñanza de las Matemáticas. 
Estudiar herramientas para el diseño de documentos matemáticos que permitan el grado de interactividad deseado por lo profesores en su transmisión a los alumnos, concretamente la utilización de MathML (XML) y HotEqn para la presentación y manipulación de la notación matemática.

Usar herramientas de tutorización y evaluación a distancia de los alumnos, que diseñen cuestionarios de auto-evaluación, listas de ejercicios, exámenes, etc. (véase sistema SIETTE : http://www.lcc.uma.es/siette).

Adaptación de los contenidos del foro a la plataforma Moodle, dado que la Universidad de Málaga recomienda utilizarla para el albergue de asignaturas.

(c) Ediciones Universidad de Salamanca 